\title{
Aprendizaje basado en problemas y el aula invertida como estrategia de aprendizaje para el fortalecimiento de competencias matemáticas ${ }^{1}$
}

\section{$\overline{\text { Problem-based learning and the Inverted Classroom }}$ as a learning strategy for the development of maths}

DOI: http://dx.doi.org/10.17981/cultedusoc.9.3.2018.0

Artículo de investigación. Fecha de recepción: 15/06/2018. Fecha de aceptación: 27/11/2018

Antonio Pava Herrera ${ }^{2}$;

Adolfo Florian Aconcha; Ana Hernández Pamplona; Aracelly Mercado Ardila; Armando Guerra Ferreira; Belisario Acosta Alfaro; Betty Salcedo Alvear; Carmenza Terraza Pava; Daisy Florian Martinez; Dora Barraza Paba; Eusebio Quintero Moreno; Francisco Miranda Alfaro; Heidys Torres Muñoz; Luis Armesto Villegas; Luz Perez De Meriño; Mabel Saucedo Paba; Ulfran Camacho Pineda; Valentina Saucedo Pava; Victor Guerra Bagarozza; Yina Rodriguez Ribon y Jolfran Silva Echavez ${ }^{3}$

IED Rural San Pedro Apóstol (Colombia) institucionPedroapostol1234@gmail.com

Para citar este artículo:

Pava, A., Florian, A., Hernández, A., Mercado, A., Guerra, A., Acosta, B., Salcedo, B., Terraza, C., Florian, D., Barraza, D., Quintero, E., Miranda, F., Torres, H., Armesto, L., Perez, L., Saucedo, M., Camacho, U., Saucedo, V., Guerra, V., Rodriguez, Y. y Silva, J. (2018). Aprendizaje basado en problemas y el aula invertida como estrategia de aprendizaje para el fortalecimiento de competencias matemáticas. Cultura. Educación y Sociedad 9(3), 35-42. DOI: http://dx.doi.org/10.17981/cultedusoc.9.3.2018.0

\section{Resumen}

Todo cambio en el ámbito educativo debe originarse con la identificación y planteamiento de problemas pedagógicos para lograr mejorar la calidad académica de los docentes y de los demás actores involucrados. El objetivo del proyecto fue realizar un diagnóstico de las competencias matemáticas por medio de las Pruebas Saber de $3^{\circ}$ para diseñar estrategias basadas en el ABP y el aula invertida como herramientas que faciliten el aprendizaje y favorezcan la motivación y concentración en esta asignatura específica. El estudio fue de tipo descriptivo. La población estuvo conformada por los estudiantes de grado tercero de la Institución Educativa Departamental (IED) Rural San Pedro Apóstol, donde se realizó un análisis de los resultados de sus pruebas, evidenciando debilidad en los planteamientos e interpretaciones de problemas matemáticos. Por tal motivo, se hizo necesaria la implementación de estas dos herramientas metodológicas para mejorar la capacidad de razonamiento y acentuar un sólido pensamiento crítico en los estudiantes.

Palabras clave: Aprendizaje basado en problemas, Aula invertida, Competencias matemáticas.

\section{Abstract}

Any change in the educational field must originate with the identification and approach of pedagogical problems to achieve the academic quality of the teachers and the other actors involved. The objective of the project was to make a diagnosis of the mathematical competences through the Knowledge Saber of $3^{\circ}$ to design strategies based on the PBL and the Inverted Classroom as tools that facilitate learning and encourage motivation and concentration in this specific subject. The study was descriptive. The population was conformed by the students of third degree of the IED Rural San Pedro Apóstol, where an analysis of the results of their tests was made, evidencing weakness in the approaches and interpretations of mathematical problems. For this reason, it became necessary to implement these two methodological tools to improve the reasoning capacity and accentuate a solid critical thinking in students.

Keywords: Problem-based learning, Inverted classroom, Mathematical competences. 1 Este artículo ha sido derivado del Programa de Fortalecimiento de la Cultura Ciudadana y Democrática CT+I a través de la IEP
apoyada en TIC en el Departamento de Magdalena: CICLON

2 Líder del grupo de investigación "Interesados por el futuro".

3 Docentes del IED Rural San Pedro Apóstol.

- The author; licensee Universidad de la Costa - CUC 


\section{Introducción}

El desarrollo social, científico, técnico y económico hoy en día requiere un aprendizaje diferente, superar el enfoque basado en la transmisión del conocimiento acumulado, en el que los estudiantes aprendían los fundamentos de un tema o materia, porque en estos momentos no resulta suficiente. El aprendizaje basado en problemas (ABP), constituye una estrategia alternativa y muy completa, porque permite integrar la adquisición de conocimientos con el aprendizaje de competencias (Vizcarro \& Juárez, 2014). En efecto, en este trabajo, mediante el ABP los estudiantes adquieren conocimientos al tiempo que aprenden a aprender de forma progresivamente independiente, también a aplicar los nuevos conocimientos en la resolución de distintos problemas similares a los que se les presentarán en el desempeño de distintas facetas de su trabajo (Vizcarro \& Juárez, 2014) (Reina, Gómez, Felizzola y Hualpa, 2016).

Se plantea esta propuesta para que no tengan cabida las inconsistencias en el aprendizaje del ser humano sintiéndose reprimidos a nivel global (en todos los aspectos) como lo cita el autor:

Cruz (2016) diciendo que la represión ha sido una constante en la historia reciente de Colombia y, en buena medida, se explica por el hecho de que el conflicto social y armado está todavía atravesado por los marcos de interpretación propios de la Guerra Fría, en donde la protesta social por lo regular se asume como otra expresión del denominado "enemigo interno", los cuales tomaron un aire renovado bajo la política de seguridad democrática del gobierno Uribe. (p. 56)

El ABP es un procedimiento didáctico, que tienen su fundamento en los enfoques de la Pedagogía activa y particularmente en la estrategia de la enseñanza denominada aprendizaje por descubrimiento y construcción, que se contrapone a la estrategia expositiva o magistral (Restrepo, 2017). En base a esto, el ABP permite desarrollar la práctica basada en la evidencia y el ejercicio de un pensamiento crítico. Esta estrategia, utilizando además los mapas conceptuales, aumenta las habilidades de pensamiento crítico y disposición personal al aprendizaje autónomo o autodirigido, porque mejora en la capacidad de razonamiento, interacción con grupos y participación activa (Saavedra; Illesca y Cabezas, 2014).

Por otra parte, el aula invertida es un modelo pedagógico que consiste en invertir los dos momentos que intervienen en la educación tradicional: el primer momento que corresponde a las actividades propias de la clase como la exposición de los contenidos por parte del docente y, el segundo, a la realización de las actividades fuera de la escuela, como las tareas (Merla y Yáñez, 2016).

Por tal motivo, la Institución Educativa Departamental Rural, en adelante IED, San Pedro Apóstol, ubicada en el corregimiento de Las Flores, Municipio de Guamal, Magdalena, en el marco del programa CICLON, cuyo interés en la construcción de una cultura ciudadana en ciencia, tecnología e innovación promoviendo en estudiantes y maestros del Departamento de Magdalena la conformación de comunidades, la realización de investigaciones que busquen la solución de problemas de su entorno y el desarrollo de sus capacidades, habilidades y competencias científicas, tecnológicas, de innovación, sociales, cognitivas y comunicativas. Este programa genera, a través de un grupo de docentes interdis- 
ciplinarios, la propuesta investigativa que vincula el ABP y el Aula Invertida como estrategias de aprendizaje para el fortalecimiento de competencias matemáticas; propuesta investigativa que nace a partir de la necesidad institucional de mejorar la calidad educativa, que se ve afectada por la falta de concentración y comprensión de los estudiantes, debido a que muchos de ellos viven en condiciones económicas precarias que conllevan a iniciar y finalizar la jornada escolar sin haberse alimentado. Sumado a eso, un rasgo de esta realidad es que el cuerpo docente no cuenta con el material de apoyo y herramientas necesarias para realizar una buena práctica pedagógica en el aula de clases.

Por tal motivo, el proyecto pretende favorecer en los estudiantes de la IED Rural San Pedro Apóstol, una participación activa por medio de la $\mathrm{ABP}$ y el Aula Invertida, la cual se plantea como una ruta posible para encontrar soluciones óptimas en el desarrollo de competencias, es decir que sean capaces de identificar, interpretar y proponer soluciones matemáticas con el fin de seleccionar las ideas y procesos que contribuyan al mejoramiento de su aprendizaje.

\section{Metodología}

Para desarrollar este trabajo de intervención se utilizó la metodología descriptiva, porque permite caracterizar la realidad, contextualizar el ambiente o entorno, interpretarlo y entrar en detalles de las experiencias que se estudian. Aporta un punto de vista "fresco, natural y holístico" de los fenómenos; es la realidad cara cara, desde la postura del que lo vive, desde su mirada y su construcción personal (Vera; Reyes y Robles, 2017).

\section{Instrumentos}

Para la realización del estudio, se analizaron por medio de comparaciones descriptivas los resultados de las pruebas saber del 2016 (tabla 1), de los estudiantes de tercero de la IED Rural San Pedro Apóstol, las Flores, con el fin de:

- Conocer el estado de las competencias de los estudiantes de la sede educativa Centro Educativo Rural las Flores y en cada etapa de los niveles educativos oficial, evaluados por el estado Nacional en el área de matemáticas.

- Tener una información específica sobre los resultados de los estudiantes de las dos sedes educativas beneficiadas con el programa Ciclón en el área de matemática.

- Para lograr el análisis descriptivo se tuvo en cuenta la definición de los siguientes niveles de desempeño (ICFES, 2016):

TABLA 1

Niveles de Desempeño del ICFES.

\begin{tabular}{|c|c|c|}
\hline Valoración & Puntuación & Concepto \\
\hline Avanzado & $(356-500)$ & $\begin{array}{l}\text { Desempeño } \\
\text { sobresaliente }\end{array}$ \\
\hline Satisfactorio & $(295-355)$ & $\begin{array}{l}\text { Adecuado } \\
\text { desempeño }\end{array}$ \\
\hline Mínimo & $(233-294)$ & $\begin{array}{l}\text { Mínimo } \\
\text { desempeño }\end{array}$ \\
\hline Insuficiente & $(100-232)$ & $\begin{array}{l}\text { Desempeño } \\
\text { insuficiente }\end{array}$ \\
\hline
\end{tabular}

Fuente: ICFES (2016).

\section{Estrategia Metodológica del ABP}

De acuerdo con Morales y Landa (2004), la ruta desarrollada para el proceso $\mathrm{ABP}$ se pudo sintetizar como se muestra a continuación en la figura 1 . 


\section{Leer y analizar el escenario del problema}

\section{Realizar lluvia de ideas}

3. Hacer una lista de aquello que se conocen

4. Hacer una lista de aquello que se desconocen

5. Hacer una lista de aquello que necesita hacerse para resolver el problema

\section{Definir el problema}

\section{Obtener información}

\section{Presentar resultados}

Figura 1. Ruta para el Desarrollo del proceso ABP.

Fuente: Morales y Landa (2004).

TABLA 2

Actividades Proyecto ABP Ejecutado en la IED Rural.

\begin{tabular}{|c|c|}
\hline Actividades & Tareas \\
\hline $\begin{array}{l}\text { 1. Docentes diseñan evaluaciones y organizan } \\
\text { talleres. }\end{array}$ & $\begin{array}{l}\text { - Organizar el trabajo de los docentes. } \\
\text { - Diseño de pautas de evaluación: } \\
\text { heteroevaluación, coevaluación y } \\
\text { autoevaluación. }\end{array}$ \\
\hline 2. Reunión con los docentes, decano. & - Presentación del Proyecto. \\
\hline $\begin{array}{l}\text { 3. Reuniones de capacitación a docentes de la } \\
\text { carrera de Educación Diferencial en cada una } \\
\text { de sus sedes. }\end{array}$ & $\begin{array}{l}\text { - Presentación del proyecto. } \\
\text { - Planificación de reuniones de capacitación. }\end{array}$ \\
\hline $\begin{array}{l}\text { 4. Presentación de la calendarización y } \\
\text { organización de los talleres. }\end{array}$ & - Acopio de la información. \\
\hline 5. Organización de los talleres tutoriales. & $\begin{array}{l}\text { - Determinar los dos docentes que participarán } \\
\text { en los talleres tutoriales. } \\
\text { - Distribución de los grupos para trabajo en los } \\
\text { talleres tutoriales. }\end{array}$ \\
\hline $\begin{array}{l}\text { 6. Implementación y ejecución de los talleres } \\
\text { con los estudiantes de } 1^{\circ} \text { año de la Carrera de } \\
\text { Pedagogía en Educación Diferencial. }\end{array}$ & $\begin{array}{l}\text { - Realización de los Talleres con los } \\
\text { estudiantes de } 1^{\circ} \text { año. } \\
\text { - Aplicación de pautas de evaluación. }\end{array}$ \\
\hline $\begin{array}{l}\text { 7. Reuniones de los docentes, análisis } \\
\text { y evaluación del logro de productos y } \\
\text { consecución de objetivos del proyecto. }\end{array}$ & $\begin{array}{l}\text { - Organización de reunión. } \\
\text { - Aplicación pauta de evaluación del taller } \\
\text { Aprendizaje basado en problemas. }\end{array}$ \\
\hline
\end{tabular}

Fuente: Jofré y Contreras, 2013. 


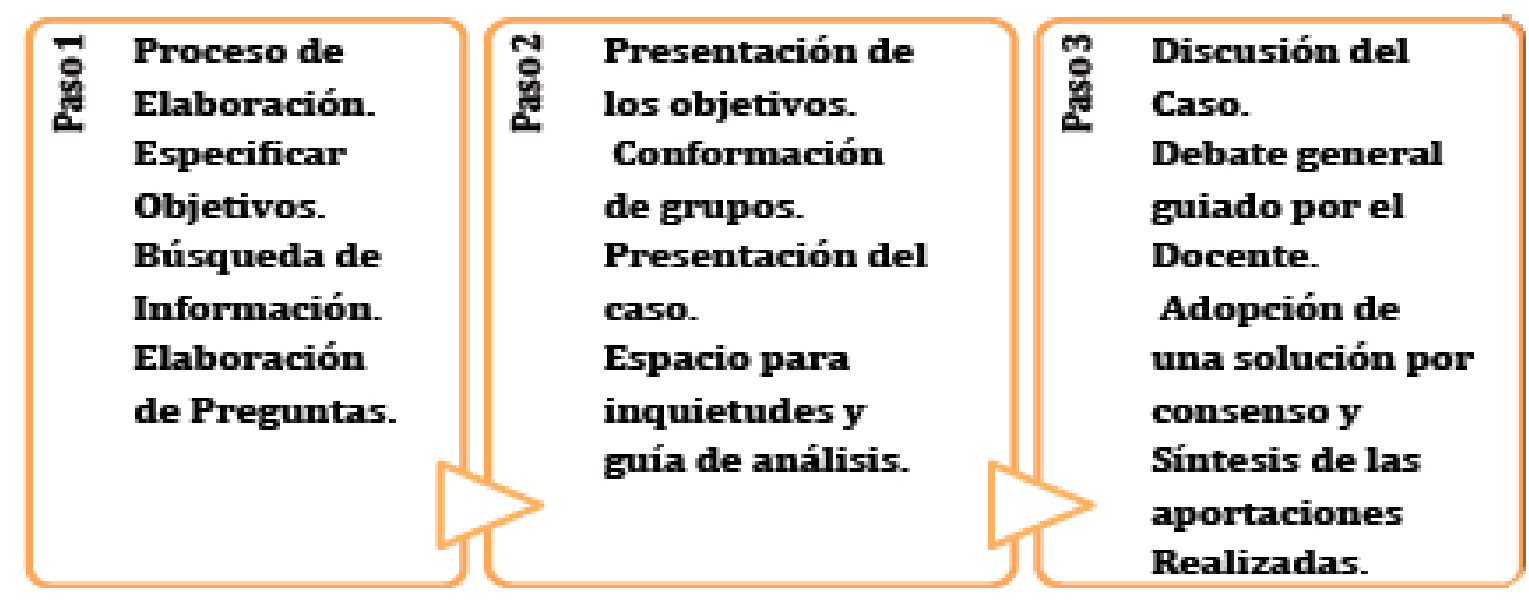

Figura 2. Esquema Resumen Construcción de Análisis de Casos.

Fuente: Jofré y Contreras, 2013.

La mayor parte de las actividades programadas se realizaron durante el primer semestre de 2018. Se organizaron talleres alrededor del diseño de los casos-problema, que fueron utilizados en el trabajo con los estudiantes. El proyecto se desarrolló con la conformación de los grupos y la elaboración del cronograma de actividades. En el proceso fue muy importante proceso de guía del docente a los estudiantes. En este acompañamiento se siguió el método $\mathrm{ABP}$, con en el análisis de casos. Estos lineamientos se resumen en la Figura 2 (Jofré y Contreras, 2013).

\section{Método Aula Invertida}

La metodología seguida en la asignatura se basó en las siguientes acciones formativas definidas por (Del Piño, Prieto, Prieto y Illeras, 2016): Video clases, actividades presenciales, estudios de casos, actividades prácticas y tutorías.

En las lecciones aplicando los videos, trabajó con los estudiantes de forma sistemática su capacidad para estudiar y aprender nuevos conceptos de forma autónoma. Después, en las clases presenciales, se planteó ciertas discusiones y cuestionamientos, a través de los cuales se descubrieron las posibles dudas y dificultades de los estudiantes, y se reforzaron los conceptos principales. Se propuso la realización de problemas aplicados a casos reales y se buscó interpelar al estudiante, proponiéndole nuevos puntos de vista para que se forme con pensamiento crítico.

\section{Valoración de la metodología del aula invertida}

El 74\% consideró que esta metodología le ha sido "bastante" o "muy" útil para comprender los conceptos, y el 39\% opinó que le ha sido "bastante" o "muy" útil para aprender a solucionar los ejercicios propuestos. El 84\% afirmó que la metodología fomenta el auto-aprendizaje con los criterios: "mucho" o "bastante". Un porcentaje del $59 \%$ prefirió en general la metodología del aula invertida, frente a las clases presenciales expositivas tradicionales (tabla 3 ). 
TABLA 3

Comparativo Aula Tradicional vs Aula Invertida

\begin{tabular}{ll}
\hline \multicolumn{1}{c}{ Aula Tradicional } & \multicolumn{1}{c}{ Aula Invertida } \\
\hline $\begin{array}{l}\text { El docente presenta } \\
\text { contenidos en la clase } \\
\text { magistral y presenta tareas } \\
\text { para ser realizadas en la } \\
\text { casa }\end{array}$ & $\begin{array}{l}\text { El docente, utilizando el aprendizaje cooperativo, guía a los } \\
\text { estudiantes, para interactuar y atender sus necesidades de } \\
\text { aprendizajes personales. }\end{array}$ \\
$\begin{array}{l}\text { A los estudiantes se le } \\
\text { transmiten contenidos } \\
\text { en clase y se le asignan } \\
\text { actividades o tareas en } \\
\text { casa. }\end{array}$ & $\begin{array}{l}\text { Se trabaja para que los estudiantes sean responsables de } \\
\text { su proceso de aprendizaje, a su ritmo y estilo. Disponen } \\
\text { fermatenidos en línea, a través de diferentes medios y } \\
\text { formaton En clase ejecutan tareas, actividades o proyectos, } \\
\text { como requieran. }\end{array}$ \\
\hline
\end{tabular}

Fuente: Merla y Yánez, 2016.

\section{Resultados}

El estudio descriptivo realizado de las Pruebas Saber (ICFES, 2016), de los estudiantes de tercer grado del IEDR San Pedro Apóstol las Flores, en cuanto a su nivel de desempeño en el área de matemáticas, arrojó un déficit en las competencias asociadas a esta, sobre todo en el Planteamiento y resolución de problemas. Esto implica que los estudiantes presentan dificultades en cuanto a la formulación de problemas y el desarrollo de estrategias para su solución mediante métodos e instrumentos válidos, así como para generalizar soluciones y estrategias para dar respuesta a nuevas situaciones problema.

De lo anterior no solo da cuenta el resultado de las pruebas saber, sino también el desempeño de los estudiantes en las clases, en las que no se desarrolla un aprendizaje significativo en función de la falta de concentración y poca motivación de estos, factor que se encuentra mediado por otras variables socioeconómicas, además de la falta de recursos pedagógicos con los que cuentan los profesores para desarrollar el currículum planteado en el marco de la asignatura.

\section{Discusión}

En las prácticas educativas se hace necesario que los estudiantes puedan trabajar a partir de estrategias que fomenten el aprendizaje significativo, sobre todo en el área matemática, siendo una de las que mayores dificultades generan, desde una propuesta integradora que permita reflexionar y poner en prácticas los conocimientos adquiridos. Esto también en función de lo planteado por el MEN (2014), en tanto propone el desarrollo de competencias matemáticas como eje transversal en la actual propuesta curricular para evaluar la calidad de la educación.

A continuación, se describen a detalle las ventajas de mayor relevancia de la metodología ABP y la de Aula Invertida:

- Ayuda a los estudiantes que tienen múltiples ocupaciones o que por diversas circunstancias no asisten a clase: el contenido principal de la clase es distribuido en línea.

- Auxilia a los estudiantes menos avanzados: la atención se dirige a los alumnos que necesitan más apoyo; no significa que los alumnos más avanzados son ignorados. 
- Ayuda a sobresalir a todos los estudiantes: la interacción docente-alumno permite aclarar dudas o resolver necesidades específicas de aprendizaje de cada alumno.

- Los estudiantes pueden trabajar a su propio paso.

- Incrementa la interacción docentealumno, alumno-alumno.

- Permite construir mejores relaciones con los discentes.

- Facilita identificar diferencias de aprendizaje reales entre los alumnos.

- El manejo de la clase es diferente en cuanto a la disciplina, los alumnos están ocupados todo el tiempo, no hay lugar para el aburrimiento.

- La plática con los padres es diferente, pues no cuestionan acerca de la conducta de su hijo que actúa respetuosamente, cumple con las tareas, entre otros. La plática va dirigida en relación a los probables problemas de aprendizaje, como asuntos personales, de salud, económicos, etc.

- Educa también a los padres, dado que el material puede ser usado por quienes así lo deseen.

- Posibilita la transparencia en cuanto a las actividades que se realizan en la escuela.

- Permite sustituir a los docentes que por diferentes situaciones se ausentan: los alumnos pueden recibir la clase del mismo docente aunque no esté presente, gracias a los materiales digitales disponibles.

\section{Referencias}

Cruz, E. (2016). El ciclo de protesta 20102016 en Colombia. Una explicación. Jurídicas CUC, 12(1), 31-62. http:// d x .doi .org/10.17981/jurid cuc.12.1.2016.3.
Del Piño, B., Prieto, B., Prieto, A., y Illeras, F. (2016). Utilización de la Metodología de Aula Invertida en una Asignatura de Fundamentos de Informática. Enseñanza y Aprendizaje de Ingeniería de Computadores, 70.

Gobernación del Magdalena (2018). Proyecto CICLÓN, Departamento del Magdalena: Ciclón. Disponible en http://programaciclon.edu.co/ciclon/ que-es-ciclon/

ICFES (2016). Guía de Interpretación y Uso de Resultados de las Pruebas Saber $3^{\circ}, 5^{\circ}$ y $9^{\circ}$. Bogotá, D.C.: Icfes.

Jofré, C. y Contreras, F. (2013). Implementación de la Metodología ABP en Estudiantes de Primer Año en la Carrera de Educación Diferencial. Estudios Pedagógicos, 102.

Ministerio de Educación Nacional (MEN), (2014). Plan de Desarrollo Sectorial. Plan Anticorrupción y Atención al ciudadano. Disponible en https://www.mineducacion.gov. co/1759/w3-article-337490.html

Merla, E. y Yánez, C. (2016). El Aula Invertida como Estrategia para la Mejora del Rendimiento Académico. Revista Mexicana de Bachillerato a Distancia, 74.

Morales, P. y Landa, V. (2004). Aprendizaje Basado en Problemas. Theoria, 154.

Restrepo, B. (2017). Aprendizaje Basado en Problemas (ABP). Una Innovación Didactica para la Enseñanza Universitaria. Pedagogía Universitaria, 10.

Saavedra, E.; Illesca, M. y Cabezas, M. (2014). Aprendizaje Basado en Problemas (ABP) como Estrategia para la Adqusición de Competencias Genéricas: Estudiantes de Nutrición y Dietética. Revista Chilena de Nutrición, 3. 
Vera, A., Reyes, D. y Robles, J. (2017). El Aprendizaje Basado en (ABP) como Estrategia para Favorecer el Aprendizaje en la Materia de Desarrollo Sustentable dentro del ITSPR. Congreso de Educación y Globalización, (4). Costa Rica.

Vizcarro, C. y Juárez, E. (2014). La Metodología del Aprendizaje basado en Problemas. Madrid.
Reina, M., Gómez, L., Felizzola, H. y Hualpa, A. (2016). Aprendizaje basado en problemas para la enseñanza de diseño experimental. INGE CUC, 12(2), 86-96. https://doi.org/10.17981/ingecuc.12.2.2016.09 